\title{
Evaluation of the Role of Palatal Tonsil Hypertrophy in Sleep Apnea Patients: Cephalometric Analysis
}

\author{
Tsutomu Nomura*, Satoshi Takeuchi, Taro Takanami, Shinichi Ishimoto \\ Department of Otolaryngology, JR Tokyo General Hospital, Tokyo, Japan \\ Email: "t-nomura@bc5.so-net.ne.jp
}

Received 20 March 2016; accepted 10 June 2016; published 13 June 2016

Copyright (C) 2016 by authors and Scientific Research Publishing Inc.

This work is licensed under the Creative Commons Attribution International License (CC BY). http://creativecommons.org/licenses/by/4.0/

(c) (i) Open Access

\section{Abstract}

The purpose of this research was to elucidate the relationship between tonsillar hypertrophy and Obstructive Sleep Apnea Syndrome (OSAS) in terms of cephalometric analysis, as well as to determine factors that affect OSAS severity. 25 patients were enrolled in this study. Lateral cephalograms of the subjects were taken. Disease severity was evaluated by the Apnea Hypopnea Index (AHI). Cephalometric measurements (angle between line S-N and line N-A (SNA), angle between line S-N and line N-B (SNB), distance between the Mandibular Plane and the Hyoid (MPH), posterior airway space, the width of the airway behind the base of tongue along the B-Go line (PAS), upper airway space, the distance parallel to long axis of the airway, between a horizontal plane tangent to the superior aspect of the hyoid and a horizontal plane tangent to the posterior palate (UAL), multiplication of PAS and UAL (PAS $\times$ UAL)) were analyzed and compared between the groups with and without tonsillar hypertrophy. PAS and PAS $\times$ UAL were lower in the no hypertrophy group. SNB was significantly lower in the severe apnea group than mild to moderate group in no hypertrophy group $(p=\mathbf{0 . 0 3 5})$. In conclusion, patients with tonsillar hypertrophy had severe obstruction, but they had a relatively larger pharyngeal space on cephalometric analysis. After exclusion of the tonsillar hypertrophy group, cephalometric analysis could be more effective for analyzing OSAS severity.

\section{Keywords}

Obstructive Sleep Apnea Syndrome, Cephalometric Analysis, Tonsillar Hypertrophy

\section{Introduction}

Obstructive Sleep Apnea Syndrome (OSAS) is a potentially life-threatening disorder caused by repetitive nar-

*Corresponding author.

How to cite this paper: Nomura, T., Takeuchi, S., Takanami, T. and Ishimoto, S. (2016) Evaluation of the Role of Palatal Tonsil Hypertrophy in Sleep Apnea Patients: Cephalometric Analysis. Open Journal of Stomatology, 6, 164-169. 
rowing and occlusion of the upper airway during sleep, and it has been associated with loud snoring and apnea. OSAS is considered to be a risk factor for hypertension, ischemic myocardial diseases, and cerebral vascular diseases [1] [2], and it is also considered to be a cause of traffic accidents due to daytime sleepiness [3] [4].

Especially in our company, which has many railway employees, if apnea symptoms were to happen during work hours, a serious disaster could occur.

Cephalometric analysis has been used by many authors to evaluate the severity of OSAS [5]-[9]. However, there are only some data on the relationship between OSAS severity and cephalometric analysis. Furthermore, tonsillar hypertrophy is one of the main causes of OSAS, but hypertrophy was not considered in the cephalometric analyses of many reports. In this study, the focus was on tonsillar hypertrophy, and the patients were divided into two groups, with and without tonsillar hypertrophy.

The aim of the present investigation is to study the relation between tonsillar hypertrophy and cephalometric analyses and efficacy of cephalometric analyses for OSAS severity.

\section{Materials and Methods}

1) Patients who were suspected to have obstructive sleep apnea syndrome (OSAS) based on a medical examination referring to International Classification of Sleep Disorders-Third Edition (ICSD-3) [10] referred at our company were the subjects of this study. This study was performed form Apr 2010 to Mar 2011.

The OSAS examination included the Epworth sleepiness scale (ESS) [11] and arterial oxygen saturation measured overnight with pulse oximetry. Patients who were suspected to have OSAS underwent polysomnography (PSG). After PSG, patients with an apnea hypopnea index (AHI) $\geq 5$ were diagnosed as having OSAS and referred to our department using International Classification of Sleep Disorders-Third Edition (ICSD-3). Twenty-five patients were enrolled in the study and assigned to two groups based on the presence or absence of tonsillar hypertrophy on examination using Friedman's palatine tonsil grading [12]; patients over grade III were assigned to the tonsillar hypertrophy group.

And all patient's background data including age, sex and mean AHI were recorded.

2) Cephalometric analyses

Lateral cephalograms of the subjects were taken with the Frankfort horizontal plane (FH plane) parallel to the floor and with the patient in centric occlusion. The cephalograms were traced to identify hard and soft tissue landmarks (Figure 1). The measuring points were digitized as two-dimensional coordinate values for calculation by a free software program (DentEd, Numata Clinic, Sendai, Japan).

3) Statistical analysis

Patients' background characteristics included age and BMI. Severity of disease was evaluated using the AHI from International Classification of Sleep Disorders-Third Edition (ICSD-3) as follows.

Mild: AHI 5 - 15.

Moderate: AHI 15 - 30.

Severe: AHI more than 30.

The SNA, SNB, MPH, PAS, UAL, and PAS $\times$ UAL values of cephalometric analysis were compared (Figure 1). These data were compared by the Mann-Whitney $U$ test. All statistical analyses were carried out with SPSS for Windows, version 10 (SPSS Inc., Chicago, IL, USA).

\section{Results}

\section{1) Overall analysis}

The data of all 25 patients are shown in Table 1. All patients were male.

The patients were divided into two groups based on the AHI score ( $<30$ and $\geq 30)$. An AHI score of $<30$ indicates mild and moderate OSAS, while an AHI score of $\geq 30$ indicates severe OSAS.

In terms of age, there is no difference between them.

$\mathrm{BMI}$ of $\mathrm{AHI}>30$ group is larger that of $\mathrm{AHI}<30$, but there is no difference.

SNB was lower and UAL was higher in the severe apnea group, but the differences were not significant.

There were no significant differences between the groups (Table 2).

2) Comparison of the groups with and without tonsillar hypertrophy

Age and BMI are higher in no-hypertrophy group without difference.

PAS and PAS $\times$ UAL were significantly lower in the non-hypertrophy group, p values were 0.011 and 0.009 


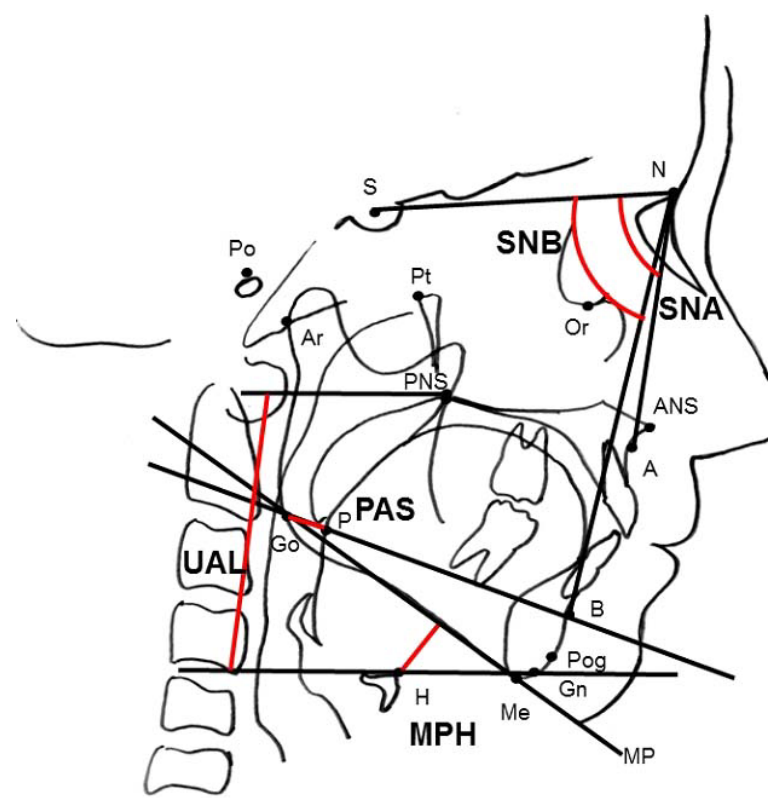

Figure 1. Cephalometric landmarks of hard and soft tissues. S: Center of the sella turcica. N: Most anterior point of the frontonasal suture. Or: Lowest point on the average left and right inferior borders of the bony orbit. Po: Highest point on the superior surface of the soft tissue of the external auditory meatus. Pt: Pterygoid point. Ar: Intersection between the external contour of the cranial base and the dorsal contour of the condylar head or neck. ANS: Apex of the anterior nasal spine. PNS: Intersection between the nasal floor and the posterior contour of the maxilla. A: Most posterior point on the anterior contour of the upper alveolar process. B: Most posterior point on the anterior contour of the lower alveolar process. Pog: Most anterior point of the contour of the chin. Gn: Point on the chin determined by bisecting the angle formed by the facial and mandibular planes. Me: Most inferior point on the mandibular symphysis. Go: Most posterior-inferior point on the convexity of the angle of the mandible. H: Most anterior-superior point on the body of the hyoid bone. P: Base of tongue on the B-Go line SNA: Angle between line S-N and line N-A. SNB: Angle between line S-N and line N-B. MP: Mandibular plane. MPH: Distance between the mandibular plane and the hyoid. PAS: Posterior airway space, the width of the airway behind the base of tongue along the B-Go line. UAL: Upper airway space, the distance parallel to long axis of the airway, between a horizontal plane tangent to the superior aspect of the hyoid and a horizontal plane tangent to the posterior palate. PAS $\times$ UAL: multiplication of PAS and UAL.

respectively (Table 3 ).

3) Non-hypertrophy group analysis

Tonsillar hypertrophy patients were excluded, and only the remaining18 patients without hypertrophy were analyzed. The patients were divided into two groups based on the AHI score $(<30$ and $\geq 30)$.

Age is same in both group. BMI is higher in AHI > 30 group, but there is no difference.

SNB was significantly lower in the severe apnea group, and PAS and PAS $\times$ UAL were lower and MPH and UAL were higher in the severe apnea group, but the differences were not significant (Table 4).

\section{Discussion}

OSAS is a sleep disorder causing excessive daytime sleepiness due to fragmentation of sleep and a chronic shortage of sleep. Napping and carelessness resulting from OSAS increases the risk of accidents. Because of the seriousness of such potential accidents, railroad companies and public transportation facilities try to prevent those caused by OSAS.

In our company, OSAS screening is performed. As part of examinations for OSAS, cephalometric analyses are often used and reported [5]-[9]. However, the efficacy of cephalometric analysis is unclear. Pracharktam et al. [5], Kim et al. [6], and Sonsuwan et al. [7] showed a relationship between MPH and the AHI. Furthermore, Susarla et al. [7], Sonsuwan et al. [8], and de Berry-Borowiecki et al. [9] emphasized the importance of PAS.

In our overall analysis (Table 1), there is no influencing factor for severity of OSAS. We suspected that tonsil state could affect cephalometric analysis. Comparison between hypertrophy of palatal tonsil group and nonhypertrophic group was performed. 
Table 1. Findings and Cephalometric score.

\begin{tabular}{|c|c|c|c|c|c|c|c|c|c|c|}
\hline Case & $\begin{array}{c}\text { Tonsil } \\
\text { hypertrophy }\end{array}$ & $\begin{array}{c}\text { Age } \\
\text { year old }\end{array}$ & AHI & BMI & SNA & SNB & PAS & MPH & UAL & $\begin{array}{c}\text { PAS } \times \\
\text { UAL }\end{array}$ \\
\hline 1 & - & 53 & 70.7 & 23.5 & 84.5 & 82.3 & 9.1 & 10.6 & 75.8 & 689.8 \\
\hline 2 & - & 67 & 77.3 & 24.0 & 73.1 & 71.8 & 7.1 & 21.4 & 87.1 & 618.4 \\
\hline 3 & - & 32 & 69.2 & 28.1 & 82.7 & 76.6 & 10.7 & 22.9 & 81.4 & 871.0 \\
\hline 4 & - & 49 & 77.5 & 27.7 & 81.2 & 75.9 & 7.9 & 28.6 & 85.7 & 677.0 \\
\hline 5 & - & 31 & 16.2 & 20.3 & 83.5 & 80.2 & 9.5 & 20.3 & 82.4 & 782.8 \\
\hline 6 & - & 52 & 71.4 & 23.9 & 77.5 & 73.9 & 9.7 & 18.1 & 88.9 & 862.3 \\
\hline 7 & - & 49 & 20.4 & 18.8 & 77.7 & 79.5 & 13.5 & 8.1 & 81.1 & 1094.9 \\
\hline 8 & - & 27 & 57.6 & 37.2 & 80.5 & 76.8 & 19.1 & 16.2 & 79.4 & 1516.5 \\
\hline 9 & - & 33 & 51.6 & 33.1 & 75.7 & 73.2 & 7.6 & 15.2 & 72.7 & 552.5 \\
\hline 10 & - & 28 & 45.4 & 31.7 & 80.9 & 74.8 & 12.5 & 23.4 & 89.1 & 1113.8 \\
\hline 11 & - & 64 & 78.9 & 22.0 & 86.5 & 79.3 & 9.1 & 16.7 & 81.8 & 744.4 \\
\hline 12 & - & 68 & 55.6 & 25.7 & 78.8 & 79.8 & 13.2 & 27.9 & 88.2 & 1164.2 \\
\hline 13 & - & 53 & 64.1 & 25.3 & 78.3 & 73.4 & 7.7 & 24.3 & 90.8 & 699.2 \\
\hline 14 & - & 54 & 27.7 & 24.6 & 85.3 & 85.1 & 7.6 & 21.2 & 72.7 & 552.5 \\
\hline 15 & - & 74 & 25.8 & 18.1 & 80.6 & 76.8 & 23.0 & 14.9 & 74.3 & 1708.9 \\
\hline 16 & - & 44 & 28.0 & 37.2 & 78.1 & 77.2 & 16.4 & 24.7 & 86.3 & 1415.3 \\
\hline 17 & - & 75 & 63.0 & 23.5 & 85.3 & 79.8 & 10.3 & 28.8 & 87.9 & 905.4 \\
\hline 18 & - & 48 & 23.2 & 28.4 & 86.3 & 80.2 & 10.2 & 8.5 & 74.6 & 760.9 \\
\hline 19 & + & 37 & 55.4 & 22.1 & 83.1 & 78.2 & 11.4 & 18.6 & 80.0 & 912.0 \\
\hline 20 & + & 34 & 94.5 & 28.7 & 88.7 & 83.4 & 27.9 & 19.1 & 77.9 & 2173.4 \\
\hline 21 & + & 28 & 68.2 & 25.1 & 85.3 & 85.6 & 17.8 & 27.4 & 89.0 & 1584.2 \\
\hline 22 & + & 36 & 68.9 & 34.9 & 79.9 & 77.7 & 22.4 & 29.3 & 94.8 & 2123.5 \\
\hline 23 & + & 29 & 24.3 & 21.0 & 79.5 & 79.5 & 15.4 & 15.4 & 84.6 & 1302.8 \\
\hline 24 & + & 35 & 40.1 & 28.1 & 78.0 & 76.5 & 14.8 & 7.4 & 92.6 & 1370.5 \\
\hline 25 & + & 54 & 35.9 & 20.8 & 84.0 & 77.4 & 11.3 & 3.8 & 77.4 & 874.6 \\
\hline
\end{tabular}

Table 2. Overall analysis (25 case).

\begin{tabular}{|c|c|c|c|c|c|}
\hline & $<30$ & (7 cases) & $\geq 30$ & (18 cases) & $\mathrm{p}$ value \\
\hline & mean & SD & mean & SD & \\
\hline age & 47.0 & 14.0 & 45.8 & 15.1 & 0.952 \\
\hline BMI & 24.1 & 6.3 & 27.0 & 4.5 & 0.146 \\
\hline SNA & 81.6 & 3.2 & 81.3 & 3.9 & 0.952 \\
\hline SNB & 79.8 & 2.5 & 77.6 & 3.6 & 0.096 \\
\hline PAS & 13.7 & 4.8 & 12.8 & 5.5 & 0.565 \\
\hline MPH & 16.2 & 5.9 & 20.0 & 7.3 & 0.204 \\
\hline UAL & 79.4 & 5.1 & 84.5 & 6.1 & 0.074 \\
\hline $\mathrm{PAS} \times \mathrm{UAL}$ & 1088.3 & 382.8 & 1080.7 & 475.9 & 0.832 \\
\hline
\end{tabular}


Table 3. Comparison between hypertrophy of palatal tonsil group and non-hypertrophic group.

\begin{tabular}{|c|c|c|c|c|c|c|}
\hline & \multirow{2}{*}{$\begin{array}{l}\text { Hyper } \\
\text { Mean }\end{array}$} & \multirow{2}{*}{$\begin{array}{c}\text { (7 cases) } \\
\mathrm{SD}\end{array}$} & \multicolumn{2}{|c|}{ Non-hyper (18 cases) } & \multirow[t]{2}{*}{$\mathrm{p}$ value } & \\
\hline & & & Mean & SD & & \\
\hline Age & 36.1 & 8.0 & 48.6 & 15.0 & 0.102 & \\
\hline AHI & 55.3 & 22.2 & 52.3 & 21.5 & 0.904 & \\
\hline BMI & 25.8 & 4.8 & 26.3 & 5.4 & 0.904 & \\
\hline SNA & 82.6 & 3.5 & 80.3 & 3.7 & 0.431 & \\
\hline SNB & 79.8 & 3.2 & 77.3 & 3.4 & 0.238 & \\
\hline PAS & 17.3 & 5.6 & 11.5 & 4.2 & 0.011 & * \\
\hline MPH & 17.3 & 8.7 & 19.7 & 6.3 & 0.586 & \\
\hline UAL & 85.2 & 6.6 & 82.4 & 6.0 & 0.364 & \\
\hline $\mathrm{PAS} \times \mathrm{UAL}$ & 1477.3 & 483.8 & 941.5 & 329.4 & 0.009 & * \\
\hline
\end{tabular}

Table 4. Non-hypertrophy group (18 cases).

\begin{tabular}{cccccc}
\hline & $<30$ & $(6$ case $)$ & $\geq 30$ & $(12$ case $)$ & p value \\
\hline Mean & SD & Mean & SD & \\
\hline Bge & 50.0 & 12.9 & 50.1 & 16.0 & 0.815 \\
SNA & 23.6 & 6.7 & 27.1 & 4.4 & 0.325 \\
SNB & 81.9 & 3.3 & 80.4 & 3.8 & 0.542 \\
PAS & 79.8 & 2.7 & 76.5 & 3.1 & 0.035 \\
MPH & 13.4 & 5.2 & 10.3 & 3.2 & 0.241 \\
UAL & 16.3 & 6.3 & 21.2 & 5.6 & 0.134 \\
PAS $\times$ UAL & 78.6 & 5.0 & 84.1 & 5.5 & 0.068 \\
\hline
\end{tabular}

This means that subjects with tonsillar hypertrophy had severe obstruction, even though they had a relatively large pharyngeal space on cephalometric analysis. Thus, evaluation of tonsillar condition is worthwhile, since tonsillectomy may offer a cure for OSAS.

In next step, in non-hypertrophy group, influencing factor were analyzed (Table 4).

In the study, PAS was lower and MPH was higher in the severe OSAS group, however the differences were not significant. Only SNB was significantly lower in severe OSAS group.

This phenomenon is clear and reasonable, since retrognathia causes a small pharyngeal space. Although in this study we evaluated only small cases, our study could serve for future studies.

In the present study, after exclusion of subjects with tonsillar hypertrophy, cephalometric analysis became more effective. With careful evaluation of oropharyngeal findings, cephalometric analysis could be an important tool for evaluating OSAS severity.

\section{Conclusion}

Patients with tonsillar hypertrophy had severe obstruction, but they had a relatively larger pharyngeal space on cephalometric analysis. After exclusion of the tonsillar hypertrophy group, cephalometric analysis could be more effective for analyzing OSAS severity.

\section{Ethical Considerations}

The study was compiled with the rules laid down by the Declaration of Helsinki. It was explained to the patients that inclusion of their data in the study was voluntary and that confidentiality and anonymity were guaranteed. 
They were also able to withdraw from the study at any time before publication without needing to give any reason. Written informed consent was obtained from all of the participants.

\section{Competing Interests}

The authors declare that they have no competing interests.

\section{Author's Contribution}

TN treated all case and have full responsibility in this paper.

ST and TT co-operated the cases and cheeked the all literature.

SI is a chief of the Department of Otolaryngology and has a responsibility of all patients' outcome and paper publication.

\section{References}

[1] Bixler, E.O., Vgontzas, A.N., Lin, H.M., et al. (2000) Association of Hypertension and Sleep-Disordered Breathing. Archives of Internal Medicine, 160, 2289-2295. http://dx.doi.org/10.1001/archinte.160.15.2289

[2] Kieiy, J.L. and McNicholas, W.T. (2000) Cardiovascular Risk Factors in Patients with Obstructive Sleep Apnea Syndrome. European Respiratory Journal, 16, 128-133. http://dx.doi.org/10.1034/j.1399-3003.2000.16a23.x

[3] Findley, L.J., Unverzagt, M.E. and Suratt, P.M. (1998) Automobile Accidents Involving Patients with Obstructive Sleep Apnea. The American Review of Respiratory Disease, 138, 337-340. http://dx.doi.org/10.1164/ajrccm/138.2.337

[4] Strohl, K., Bonnie, R., Findly, L., et al. (1994) Sleep Apnea, Sleepiness, and Driving Risk. American Journal of Respiratory and Critical Care Medicine, 150, 1463-1473. http://dx.doi.org/10.1164/ajrccm.150.5.7952578

[5] Pracharktam, N., Nelson, S., Hans, M.G., et al. (1996) Cephalometric Assessment in Obstructive Sleep Apnea. American Journal of Orthodontics and Dentofacial Orthopedics, 109, 410-419. http://dx.doi.org/10.1016/S0889-5406(96)70123-3

[6] Kim, S.J., Kim, Y.S., Park, J.H. and Kim, S.W. (2012) Cephalometric Predictors of Therapeutic Response to Multilevel Surgery in Patients with Obstructive Sleep Apnea. Journal of Oral and Maxillofacial Surgery, 70, 1404-1412. http://dx.doi.org/10.1016/j.joms.2011.03.016

[7] Susarla, S.M., Abramson, Z.R., Dodson, T.B. and Kaban, L.B. (2010) Cephalometric Measurement of Upper Airway Length Correlates with the Presence and Severity of Obstructive Sleep Apnea. Journal of Oral and Maxillofacial Surgery, 68, 2846-2855. http://dx.doi.org/10.1016/j.joms.2010.06.196

[8] Sonsuwan, N., Suchachaisri, S. and Chaloeykitti, L. (2011) The Relationships between Cephalometric Parameters and Severity of Obstructive Sleep Apnea. Auris Nasus Larynx, 38, 83-87. http://dx.doi.org/10.1016/j.anl.2010.04.009

[9] deBerry-Borowiecki, B., Kukwa, A. and Blanks, R.H. (1988) Cephalometric Analysis for Diagnosis and Treatment of Obstructive Sleep Apnea. Laryngoscope, 98, 226-234. http://dx.doi.org/10.1288/00005537-198802000-00021

[10] Sateia, M.J. (2014) International Classification of Sleep Disorders-Third Edition: Highlights and Modifications. Chest, 146, 1387-1394. http://dx.doi.org/10.1378/chest.14-0970

[11] Johns, M.W. (1991) A New Method for Measuring Day-Time Sleepiness: The Epworth Sleepiness Scale. Sleep, 14, 540-545.

[12] Friedman, M., Ibrahim, H. and Bass, L. (2002) Clinical Staging for Sleep-Disordered Breathing. OtolaryngologyHead and Neck Surgery, 127, 13-21. http://dx.doi.org/10.1067/mhn.2002.126477 